\title{
No Difference in Early Analgesia Between Liposomal Bupivacaine Injection and Intrathecal Morphine After TKA
}

\author{
John W. Barrington MD, Roger H. Emerson MD, Scott T. Lovald PhD, \\ Adolph V. Lombardi MD, Keith R. Berend MD
}

Published online: 23 June 2016

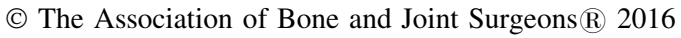

\begin{abstract}
Background Opioid analgesics have been a standard modality for postoperative pain management after total knee arthroplasty (TKA) but are also associated with increased risk of nausea, pruritus, vomiting, respiratory depression, prolonged ileus, and cognitive dysfunction.
\end{abstract}

Two of the authors (JWB, RHE) have received research support funding from Pacira Pharmaceuticals (Parsippany, NJ, USA). The institution of three of the authors (STL, AVL, KRB) has received, during the study period, funding from Pacira Pharmaceuticals. One of the authors (RHE) lists the following relevant financial activities outside of this work and/or any other relationships or activities that readers could perceive to have influenced, or that give the appearance of potentially influencing, this manuscript: Pacira Pharmaceuticals (speakers' bureau) in the amount of less than USD 10,000. One of the authors (JWB) lists the following relevant financial activities outside of this work and/or any other relationships or activities that readers could perceive to have influenced, or that give the appearance of potentially influencing, this manuscript (all consulting): Zimmer Biomet (Warsaw, IN, USA) in the amount of USD 100,001 to USD $1,000,000$, Pacira Pharmaceuticals in the amount of less than USD 10,000 , Smith \& Nephew (London, UK) in the amount of less than USD 10,000, United Surgical Partners (Addison, TX, USA) in the amount of less than USD 10,000, and Orthosensor (Dania Beach, FL, USA) in the amount of less than USD 10,000. One of the authors (STL) is an employee of Exponent (Menlo Park, CA, USA), a scientific and engineering consulting firm. Exponent has been paid fees by entities in the biomedical arena for their consulting services (amount varies by project). The institution of one or more of the authors (AVL, KRB) has received funding from Zimmer Biomet, Pacira Pharmaceuticals, Orthosensor, Stryker (Mahwah, NJ, USA), and Kinamed (Camarillo, CA, USA). One of the authors (AVL) lists the following relevant financial activities, during the study period, outside of this work and/or any other relationships or activities that readers could perceive to have influenced, or that give the appearance of potentially influencing, this manuscript: an amount of more than USD 1,000,001 from Zimmer Biomet (royalties, consulting fees), an amount of USD 10,000 to USD 100,000 from Innomed (royalties; Savannah, GA, USA), an amount of less than USD 10,000 from Pacira Pharmaceuticals (consulting fees), and an amount of less than
There is still a need for a method of anesthesia that can deliver effective long-term postoperative pain relief without incurring the high cost and health burden of opioids and nerve blocks.

Questions/purposes (1) Is liposomal bupivacaine-based periarticular injection (PAI) more effective than morphinebased spinal anesthesia or ropivacaine-based PAI in

USD 10,000 from Orthosensor (consulting fees, royalties). One of the authors certifies that he (KRB) has or may receive payments or benefits, during the study period, an amount of more than USD 1,000,001 from Zimmer Biomet (consulting fees, royalties). All ICMJE Conflict of Interest Forms for authors and Clinical Orthopaedics and Related Research ${ }^{\mathbb{R}}$ editors and board members are on file with the publication and can be viewed on request.

Clinical Orthopaedics and Related Research ${ }^{\mathbb{R}}$ neither advocates nor endorses the use of any treatment, drug, or device. Readers are encouraged to always seek additional information, including FDAapproval status, of any drug or device prior to clinical use.

Each author certifies that his institution approved the human protocol for this investigation, that all investigations were conducted in conformity with ethical principles of research, and that informed consent for participation in the study was obtained.

This work was performed at Plano Orthopedic Sports Medicine and Spine Center, Plano, TX, USA, and at Joint Implant Surgeons, New Albany, OH, USA.

\section{J. W. Barrington}

Plano Orthopedic Sports Medicine and Spine Center, Plano, TX, USA

R. H. Emerson

Texas Center for Joint Replacement, Plano, TX, USA

S. T. Lovald ( $\square)$

Exponent, Inc, 149 Commonwealth Drive, Menlo Park, CA

19104, USA

e-mail: slovald@exponent.com

A. V. Lombardi, K. R. Berend

Joint Implant Surgeons, New Albany, OH, USA 
controlling postoperative pain after TKA? (2) Do patients treated with liposomal bupivacaine-based PAI experience fewer opioid-related adverse events compared with patients treated with morphine-based spinal anesthesia or ropivacaine-based PAI in controlling postoperative pain after TKA?

Methods This multicenter, blind trial randomized 119 patients undergoing TKA with spinal anesthesia to receive spinal anesthesia plus periarticular injection with liposomal bupivacaine (40 patients), spinal anesthesia with bupivacaine plus intrathecal morphine (41 patients) but no liposomal bupivacaine injection, or spinal anesthesia with bupivacaine (38 patients) and no liposomal bupivacaine injection. The two groups that did not receive periarticular liposomal bupivacaine did receive periarticular injection with ropivacaine, and all three groups had ketorolac (30 $\mathrm{mg})$ plus epinephrine $(1: 1000)$ in the periarticular injections. Patients in all three groups received identical perioperative multimodal analgesic and antiemetic drugs. All patients were analyzed in the group to which they were randomized and no patients were lost to followup. The primary study endpoints were visual analog score (VAS) for pain and narcotic use during postoperative day 1 . Secondary endpoints included side effects associated with narcotic administration during the hospital stay.

Results Mean VAS pain in the liposomal bupivacaine PAI group was lower than that for the ropivacaine PAI group at 6 hours $(1.8 \pm 2.1$ versus $3.3 \pm 2.3, \mathrm{p}=0.005$, mean difference: $1.5,95 \%$ confidence interval [CI], 0.5$2.5)$ and 12 hours $(1.5 \pm 2.0$ versus $3.3 \pm 2.4, \mathrm{p}<0.001$, mean difference: $1.8,95 \% \mathrm{CI}, 0.8-2.8)$ after surgery. The morphine spinal group had lower pain compared with the liposomal bupivacaine PAI group at 6 hours $(0.9 \pm 1.8$ versus $1.8 \pm 2.1, \mathrm{p}=0.035$, mean difference: $1.0,95 \% \mathrm{CI}$, $0.1-1.8)$, but there was no difference at 12 hours $(0.8 \pm 1.5$ versus $1.5 \pm 2.0, \mathrm{p}=0.086$, mean difference: $0.7,95 \% \mathrm{CI}$, -0.1 to 1.5 ). The magnitude of the differences at 6 and 12 hours are near the lower end of minimal clinically important differences reported in the literature, and thus the improvement shown in this study may only represent a small clinical improvement. Both the liposomal bupivacaine group (13\% [five of 40]) and the ropivacaine group (5\% [two of 38]) had fewer incidents of itching (pruritus) than the spinal morphine group (38\% [15 of 41]) $(\mathrm{p}=$ $0.001)$.

Conclusions This prospective multicenter three-arm blind randomized controlled trial showed potentially improved pain control at 6 and 12 hours in the liposomal bupivacaine and intrathecal morphine groups compared with the ropivacaine group at the cost of much higher incidences of pruritus (itching) in the intrathecal morphine group. Based on these results, we prefer the use of PAI with liposomal bupivacaine as an alternative to spinal anesthesia with intrathecal morphine as a result of similar postoperative pain control and the potential for reducing adverse events. Level of Evidence Level I, therapeutic study.

\section{Introduction}

More than 1.1 million total joint arthroplasties (TJAs) are performed annually in the United States [39], over 700,000 of which are primary TKAs. Despite its success improving the quality of life of patients with arthritis [31], pain after TKA can sometimes be severe and difficult to control [1]. Clinical studies have shown that severe postoperative pain can be associated with an increased risk of complications, including rehabilitation delay [59], prolonged return to normal functioning $[27,28]$, progression to persistent pain states [46, 68], prolonged hospital stay [49], and increased readmission rate [21], all of which can lead to increased cost of care [4, 42, 62, 69].

Multiple anesthesia and analgesia modalities have been proposed and used over the past decade with an emphasis on rapid recovery and improve outcomes. Unfortunately, many complications after TKA may be associated with pain management strategies themselves. Opioid analgesics, including intravenous, patient-controlled, and oral, have been a standard modality for postoperative pain management but are also associated with increased risk of nausea, pruritus, vomiting, respiratory depression, prolonged ileus, and cognitive dysfunction [18, 64, 85]. Regional pain control techniques such as femoral nerve blockade may limit exposure to opioid-related adverse events but may lead to other complications such as quadriceps weakness, neuropathy, and postoperative falls $[8,75]$.

There is still a need for a method of anesthesia that can deliver effective postoperative pain relief without incurring the high cost and health burden of opioids and nerve blocks. A periarticular injection (PAI) administered at the end of the surgical procedure has been shown to decrease pain, increase function, and reduce opioid-related adverse events after TJA in both case series and randomized controlled trials $[15,48,66]$. Published studies have suggested that PAI is more cost-efficient and easier to perform than other regional modalities such as femoral neck blocks $[1,54]$.

PAI cocktails may include local anesthetics along with various additions such as nonsteroidal antiinflammatory drugs and epinephrine. They also can contain antibiotics, steroids, or other locally active agents. These cocktails have been shown to be effective in the acute perioperative period but have finite periods of action. Traditional amide local anesthetics such as bupivacaine or ropivacaine (which is reported to have a faster onset than bupivacaine but with a similar duration of action $[2,7,11,12,22,25,37$, $41,50,52,55,58,74,86]$ ) have been the mainstay of most 
TKA PAI cocktails. In addition to amide local anesthetics, some investigators have chosen to also include intrathecal morphine as part of their pain management protocol. A single-dose local analgesic has been introduced that delivers bupivacaine over time (Exparel; Pacira Pharmaceuticals, Parsippany, NJ, USA). This formulation has been associated with decreased postoperative pain in patients undergoing TJA [6]. However, early comparative studies either had major methodological flaws [5] or were adequately powered but lacked randomization or anesthesia/ injection protocol standardization [6]. We therefore undertook a multicenter, double-blind, three-arm, randomized controlled trial (RCT) to investigate the effectiveness of a liposomal bupivacaine-based PAI (Exparel) versus ropivacaine-based (Naropin; Fresenius Kabi USA, Lake Zurich, IL, USA) PAI with a third arm that included a morphine-based spinal (Duramorph; West-Ward Pharmaceuticals, Eatontown, NJ, USA) in controlling postoperative pain in patients undergoing TJA.

Specifically, we asked: (1) Is liposomal bupivacainebased PAI more effective than morphine-based spinal anesthesia or ropivacaine-based PAI in controlling postoperative pain after TKA? (2) Do patients treated with liposomal bupivacaine-based PAI experience fewer opioidrelated adverse events compared with patients treated with morphine-based spinal anesthesia or ropivacaine-based PAI in controlling postoperative pain after TKA?

\section{Patients and Methods}

This study was a prospective, multicenter (Columbus, OH, USA, and Plano, TX, USA), double-blind (patients and data-recording research teams were blinded to the identity of the anesthetic), three-arm, RCT of patients undergoing primary unilateral TKA. Patients were recruited between November 2013 and October 2015. Five surgeons at the two centers (three surgeons in Ohio, two surgeons in Texas) provided treatment for the enrolled patients. The study was approved by the institutional review board at each respective facility.

To be considered for the study, subjects had to be at least 18 years of age, be willing and able to complete surveys, and sign a consent form approved by the institutional review board of the participating centers. Patients with severe hepatic or renal impairment were excluded from participation. Patients with lower back conditions, which could potentially complicate the administration of the spinal anesthesia, were also excluded. One hundred nineteen patients with 119 TKAs were enrolled in the study and randomized into the three investigated groups. Patients in the first group (40 patients) were given a bupivacaine spinal and periarticular injections with liposomal bupivacaine (Exparel), bupivacaine, ketorolac, and epinephrine (Table 1). Hereafter this group is referred to as the "liposomal bupivacaine PAI" group. Patients in the second group (41 patients) were given a spinal with bupivacaine and intrathecal morphine and periarticular injections with ropivacaine, ketorolac, and epinephrine. Hereafter this group is referred to as the "morphine spinal" group. Patients in the third group (38 patients) were given a bupivacaine spinal and periarticular injections with ropivacaine, ketorolac, and epinephrine. Hereafter this group is referred to as the "ropivacaine PAI" group.

Subject randomization was completed per CONSORT guidelines [57] using the "Random Sequence Generator" from www.random.org to provide a random order list. Initially, randomization was set for a sample size of 50, after which the appropriate sample size was determined using power analysis. Patients were enrolled in each group until the minimum sample size was achieved for all three groups.

An initial power analysis was performed to determine the number of patients required to show a minimally clinically important difference (MCID) of 1.3 in visual analog scale (VAS) scores between groups. This difference is at the lower end of the range of MCIDs reported in the literature $[13,32,33,56,73,81,82]$ and thus the sample

Table 1. Drug regimen for each of the three treatment groups involved in the study

\begin{tabular}{|c|c|c|c|}
\hline Analgesic modality & Group 1 (liposomal bupivacaine*) & Group 2 (intrathecal morphine ${ }^{\dagger}$ ) & Group 3 (ropivacaine $\mathrm{Hcl}^{+}$) \\
\hline Spinal anesthesia & $1.2 \mathrm{~mL}$ of $0.75 \%$ bupivacaine ( $9 \mathrm{mg}$ ) & $\begin{array}{l}1.2 \mathrm{~mL} \text { of } 0.75 \% \text { bupivacaine }(9 \mathrm{mg}) \\
0.2-0.25 \mathrm{mg} \text { intrathecal morphine }\end{array}$ & $1.2 \mathrm{~mL}$ of $0.75 \%$ bupivacaine $(9 \mathrm{mg})$ \\
\hline \multirow[t]{4}{*}{$\begin{array}{l}\text { Periarticular injection } \\
\text { cocktail }\end{array}$} & $\begin{array}{l}20 \mathrm{~mL} \text { of } 1.3 \% \text { liposomal bupivacaine } \\
25 \mathrm{~mL} \text { of } 0.5 \% \text { bupivacaine }\end{array}$ & $50 \mathrm{~mL}$ of $0.5 \%$ ropivacaine & $50 \mathrm{~mL}$ of $0.5 \%$ ropivacaine \\
\hline & $30 \mathrm{mg}$ ketorolac & $30 \mathrm{mg}$ ketorolac & $30 \mathrm{mg}$ ketorolac \\
\hline & $1 \mathrm{mg}$ of $1: 1000$ epinephrine & $1 \mathrm{mg}$ of $1: 1000$ epinephrine & $1 \mathrm{mg}$ of $1: 1000$ epinephrine \\
\hline & $\begin{array}{l}\text { Normal saline to make } 60 \mathrm{~mL} \text { total } \\
\text { injectate }\end{array}$ & $\begin{array}{l}\text { Normal saline to make } 60 \mathrm{~mL} \text { total } \\
\text { injectate }\end{array}$ & $\begin{array}{l}\text { Normal saline to make } 60 \mathrm{~mL} \text { total } \\
\text { injectate }\end{array}$ \\
\hline
\end{tabular}

* Exparel, Pacira Pharmaceuticals, Parsippany, NJ, USA; ${ }^{\dagger}$ Duramorph, West-Ward Pharmaceuticals, Eatontown, NJ, USA; ${ }^{\star}$ Naropin, Fresenius Kabi USA, Lake Zurich, IL, USA. 
size would be expected to ensure the ability to observe differences that are clinically meaningful. The calculation assumed an $80 \%$ power at an $\alpha$ level of 0.05 . Using a primary endpoint of pain at 1 day postoperatively, the analysis determined that 38 patients were required to be enrolled in each group (assuming a SD of 2). Although the initial power analysis is only valid for two groups, we conducted a post hoc analysis for three groups to determine the number of patients required to show a Cohen's effect size, $f$, of 0.30 ( $80 \%$ power at an $\alpha$ level of 0.05$)$. The analysis determined a similar number of patients required for each treatment group $(n=37)$. Therefore, the larger sample size of 38 patients per group was selected to meet both requirements.

The PAI technique used a 22-G, moving-needle, multisite injection technique. Both spinal anesthesia and PAI techniques were standardized at both sites and among surgeons. For the PAI technique in particular, surgeons were trained by use of an educational video demonstrating the technique, location, and amount for injection. The technique included injecting the entire capsule with approximately 50 injections, including the quadriceps and subcutaneous layer. Injecting near the midline was avoided. All patients received standardized, preemptive multimodal perioperative analgesia (Table 2). Postoperative opioid administration was done only on an as-needed basis. Patients were mobilized with the assistance of a physical therapist on the day of surgery, when possible. The inpatient rehabilitation program included isometric exercises, active and active-assisted knee ROM (without a continuous passive motion machine), and assisted gait progressing to walking as tolerated by the patient. American Association of Orthopaedic Surgeons guidelines were followed for risk-stratified venous thromboembolism (VTE) prophylaxis [71]. A majority of patients in the study received multimodal VTE prophylaxis with mechanical compression devices and aspirin.

The groups did not differ in regard to age, body mass index, or sex (Table 3). Sixty-seven percent of the patients were female ( 80 of 119 ) and $33 \%$ were male (39 of 119). There were no differences in terms of preoperative opioid use for patients between groups (liposomal bupivacaine PAI: $38 \%$ [15 of 40], morphine spinal: $39 \%$ [16 of 41], ropivacaine PAI: $24 \%$ [nine of 38 ], $p=0.288$ ). In addition, there were no differences in terms of preoperative ROM, preoperative Knee Society Score (KSS), or preoperative KSS function score (Table 3).

There were no withdrawals from the study nor were any patients lost to followup (Fig. 1). All patients were analyzed in the groups to which they were randomized. Primary outcome measures included 10-point numerical, or visual analog, pain scores at rest (VAS $[29,34,40,44,47,79$, $81,84])$ at 1,6 , and 12 hours postoperatively; VAS pain scores at postoperative days 1 (at the hospital), 2, 3, 4, 5, and 6 (upon awakening in the morning); and in-hospital narcotic use (converted into morphine equivalents). Pain scores were collected both in and out of the hospital. The primary endpoint of pain at 1 day postoperatively was used to determine the appropriate sample size. Total narcotics were calculated over the full length of stay of each patient. Secondary outcome measures included day of surgery ambulation; postoperative day 1 straight leg raise and maximum activeassisted ROM; postoperative nausea/vomiting resulting in additional medications or a change in diet; complaints of itching; additional opioid-related adverse events such as constipation/obstipation, urinary retention or recatheterization, dizziness, confusion, excessive somnolence, or hypoventilation/hypoxia; and hospital length of stay. Each patient was asked about complications by the research coordinator while in the hospital and during followup calls on postoperative day 7 and day 14 . The research coordinator was not blinded to the study group.

\section{Statistical Analysis}

Statistical analysis was performed with the assistance of the Exponent, Inc team using SAS software (SAS Institute

Table 2. Standardized preemptive multimodal perioperative analgesia protocol adopted for all patients in the study

\begin{tabular}{ll}
\hline Medication & Dosage \\
\hline Celebrex* $^{*}$ & $200 \mathrm{mg}$ PO $\times 1$ preoperatively; 200 mg QD $\times 10$ days postoperatively \\
Oxycontin $^{\dagger}$ & $20 \mathrm{mg} \mathrm{PO} \times 1$ preoperatively $(10 \mathrm{mg}$ for patients $>70$ years old) \\
Tylenol $^{*}$ & $1 \mathrm{~g} \mathrm{IV} \mathrm{on} \mathrm{induction,} 4 \mathrm{mg}$ IV in the morning of postoperative day 1 \\
Decadron $^{\S}$ & $10 \mathrm{mg} \mathrm{IV}$ on induction; $4 \mathrm{mg}$ IV in the morning of postoperative day 1 \\
Zofran $^{\prime \prime}$ & $8 \mathrm{mg} \mathrm{IV}$ on induction; $4 \mathrm{mg}$ IV PRN \\
Tranexamic acid & $1 \mathrm{~g} \mathrm{IV} \mathrm{on} \mathrm{induction;} 1 \mathrm{~g}$ at 3 hours postoperatively \\
\hline
\end{tabular}

* G.D. Searle LLC Division of Pfizer Inc, New York, NY, USA; ${ }^{\dagger}$ Purdue Pharma, LP, Stamford, CT, USA; ${ }^{*}$ McNeil Consumer Healthcare, Fort Washington, PA, USA; ${ }^{\S}$ Merck \& Co, Inc, Kenilworth, NJ, USA; "GlaxoSmithKline, Brentford, UK; PO = orally; QD = per day; PRN = as needed. 
Table 3. Patient demographics with regard to age, BMI, sex, side of surgery, and preoperative functioning

\begin{tabular}{|c|c|c|c|c|}
\hline Variable & $\begin{array}{l}\text { Liposomal bupivacaine PAI } \\
\text { Mean (SD) }\end{array}$ & Morphine spinal & Ropivacaine PAI & $\mathrm{p}$ value \\
\hline Age & $69(9)$ & $69(8)$ & $69(9)$ & 0.976 \\
\hline BMI & $34(8)$ & $34(6)$ & $34(8)$ & 0.808 \\
\hline Operating room time (minutes) & $74(20)$ & $74(22)$ & $64(26)$ & 0.076 \\
\hline Preoperative ROM (degrees) & $108(14)$ & $109(14)$ & $112(11)$ & 0.496 \\
\hline Preoperative Knee Society functional score & $46(14)$ & $48(12)$ & $51(15)$ & 0.241 \\
\hline \multirow[t]{2}{*}{ Preoperative Knee Society total score } & $35(15)$ & $33(19)$ & $38(18)$ & 0.514 \\
\hline & Number & & & \\
\hline Female & $29(73 \%)$ & $25(61 \%)$ & $26(68 \%)$ & 0.534 \\
\hline Male & $11(28 \%)$ & $16(39 \%)$ & $12(32 \%)$ & \\
\hline TKA on left side & $24(60 \%)$ & $22(54 \%)$ & $18(47 \%)$ & 0.535 \\
\hline TKA on right side & $16(40 \%)$ & $19(46 \%)$ & $20(53 \%)$ & \\
\hline Preoperative opioid use (yes) & $15(38 \%)$ & $16(39 \%)$ & $9(24 \%)$ & 0.288 \\
\hline
\end{tabular}

$\mathrm{PAI}=$ periarticular injection; $\mathrm{BMI}=$ body mass index .

Inc, Cary, NC, USA) Version 9.4. Demographics, pain scores, narcotic use, complications, and length of stay were assessed for normality and analysis of variance test results are presented to compare groups for continuous variables and chi square tests for categorical variables. A Bonferroni correction was used to adjust for multiple testing, giving an $\alpha$ of $0.00185(0.05 / 27)$ to test for significance. Pairwise multiple comparisons were adjusted based on Tukey's methods. For result variables that are not normally distributed, the medians and ranges are also provided and nonparametric Kruskal-Wallis tests to compare treatment groups were applied using the Dwass, Steel, CritchlowFligner method for multiple comparisons.

\section{Results}

Mean VAS pain in the liposomal bupivacaine PAI group was lower than that for the ropivacaine PAI group at 6 hours $(1.8 \pm 2.1$ versus $3.3 \pm 2.3, \mathrm{p}=0.005$, mean difference: $1.5,95 \%$ confidence interval $[\mathrm{CI}], 0.5-2.5)$ and 12 hours $(1.5 \pm 2.0$ versus $3.3 \pm 2.4, \mathrm{p}<0.001$, mean difference: $1.8,95 \%$ CI, 0.8-2.8) after surgery (Table 4$)$. The morphine spinal group had lower pain compared with the liposomal bupivacaine PAI group at 6 hours $(0.9 \pm 1.8$ versus $1.8 \pm 2.1, \mathrm{p}=0.035$, mean difference: $1.0,95 \% \mathrm{CI}$, $0.1-1.8)$, but there was no difference at 12 hours $(0.8 \pm 1.5$ versus $1.5 \pm 2.0, \mathrm{p}=0.086$, mean difference: $0.7,95 \% \mathrm{CI}$, -0.1 to 1.5$)$. With the numbers available, there were no other differences in pain among the three groups beyond 12 hours. Median and range for all VAS scores are also presented because the outcomes were not normally distributed (Table 4). For all treatment groups, pain levels were highest at postoperative day 2; however, patients in the ropivacaine PAI group experienced an early peak in VAS scores at 6 hours followed by a plateau and a second peak at day 2 (Fig. 2). The magnitude of the differences at 6 and 12 hours are near the lower end of MCIDs reported in the literature $[13,32,33,56,73,81,82]$ and thus the improvement shown with this sample may only represent a small clinical improvement. However, it is noted that pain scores for all groups were low, near, or below 3 at postoperative day 1 , and thus it may add perspective to consider that the difference at 12 hours is $55 \%$ of the ropivacaine PAI score, well beyond the high end of MCID percentages reported in the literature $(35 \%)$.

Both the liposomal bupivacaine group (13\% [five of 40]) and the ropivacaine group (5\% [two of 38]) had fewer incidents of itching (pruritus) than the spinal morphine group (38\% [15 of 41]) ( $\mathrm{p}=0.001)$ (Table 5). Using the liposomal bupivacaine group as the reference, the odds ratio for itching in the morphine spinal group was 4.2 (95\% CI, $1.4-13.1, \mathrm{p}=0.0132$ ), whereas the odds ratio for the ropivacaine group was $0.4(95 \% \mathrm{CI}, 0.1-2.1, \mathrm{p}=0.278)$. With the numbers available, we found no difference in the incidence of postoperative nausea among the three groups $(\mathrm{p}=$ 0.445 ) (Table 5). There were also no differences in the total narcotics consumed for the liposomal bupivacaine PAI (71 $\pm 93 \mathrm{mg})$, ropivacaine PAI $(75 \pm 58 \mathrm{mg})$, and morphine spinal groups $(89 \pm 14 \mathrm{mg})(\mathrm{p}=0.910)($ Table 4$)$.

There were no differences between groups in day 1 maximum active ROM ( $\mathrm{p}=0.101)$, length of stay $(\mathrm{p}=$ $0.816)$, percentage of patients who could ambulate on day 1 $(\mathrm{p}=0.901)$, or perform a straight leg raise on day $1(\mathrm{p}=$ 0.602 ) (Table 5). There were also no differences in postoperative KSS and KSS function scores (Table 5). 
Fig. 1 The study flow diagram is shown for the three-arm randomized controlled trial. $\mathrm{I} / \mathrm{E}=$ inclusion/exclusion.

\section{Study Flow Diagram}
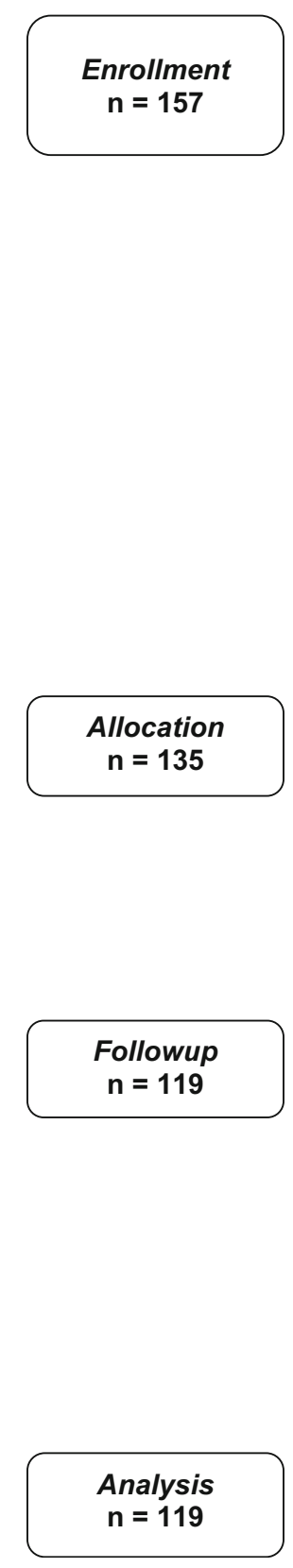

\section{Discussion}

There has been a long-term effort to reduce opioid consumption after TKA with a goal of reducing opioid-related adverse events, shortening the hospital length of stay, reducing readmission, and increasing physical therapy participation $[63,75]$. It has been proposed that liposomal bupivacaine can improve the duration of effectiveness for PAI and thus may provide sufficient analgesia while also reducing the amount of opioid medications delivered for effective pain control. The current study occurred in

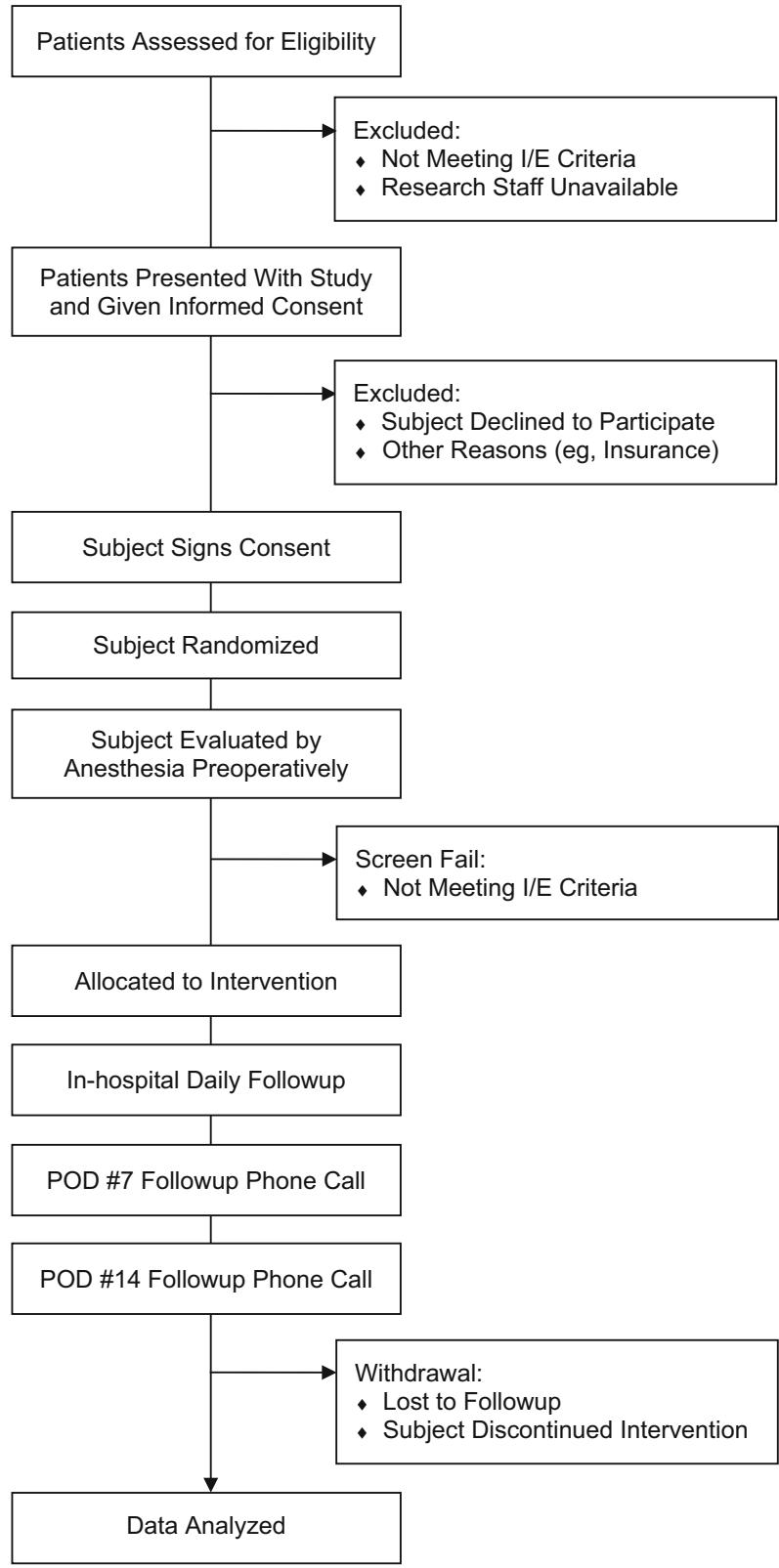

settings that already had established total joint pathways in place, including preoperative education [70], patient-focused care initiatives [3, 17, 19, 36, 43, 45, 60, 67, 87], multimodal analgesia regimens $[9,10,16,20,24,26,35$, $38,53,72,76,78,80,83]$, and postoperative rehabilitation pathways $[16,51,77]$ to focus the results on the three randomized treatment groups described. We found that the primary outcome measure of pain control was better at 6 and 12 hours postoperatively in the liposomal bupivacaine PAI and spinal morphine groups compared with the ropivacaine PAI group. However, this improvement was 


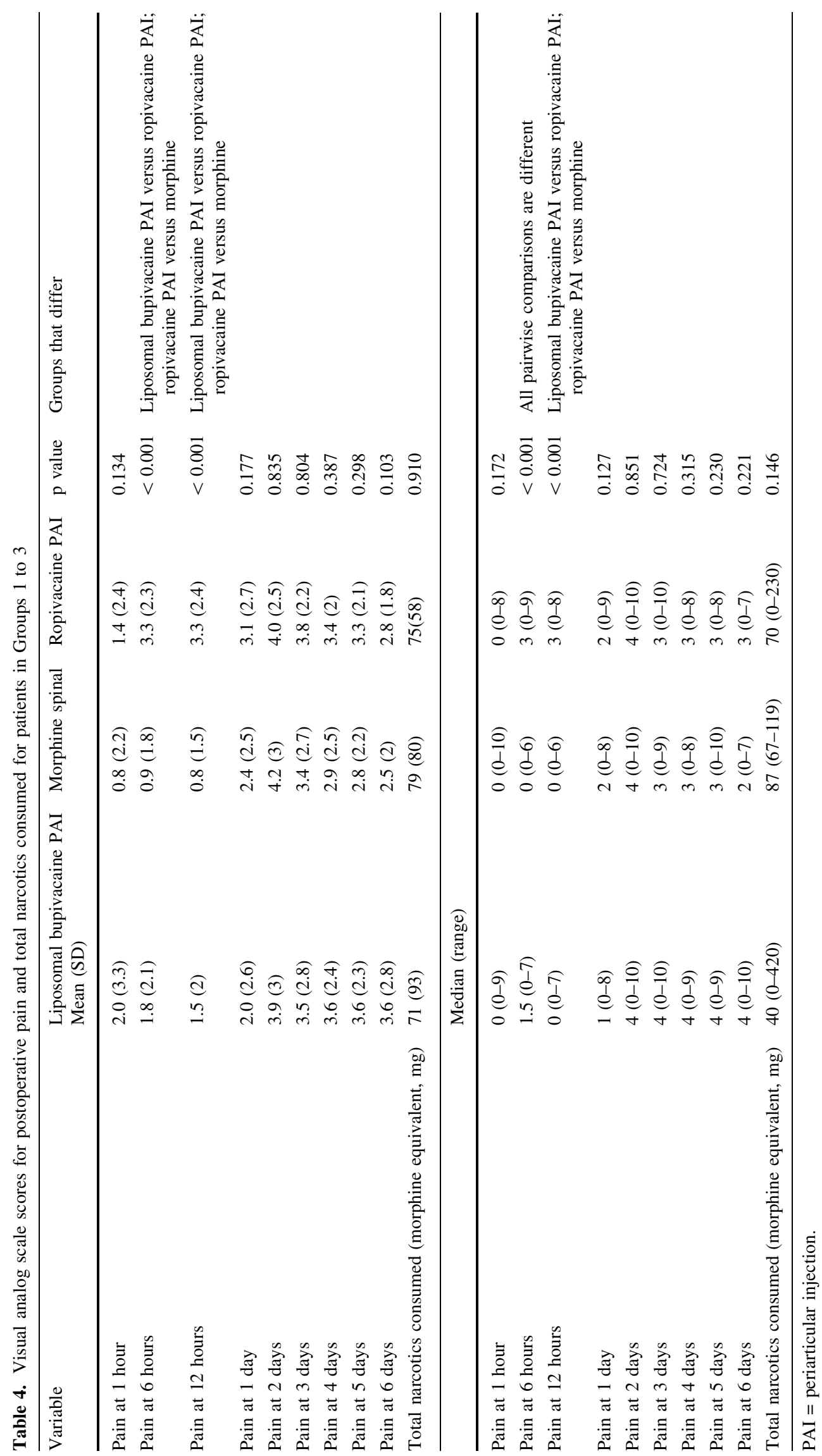




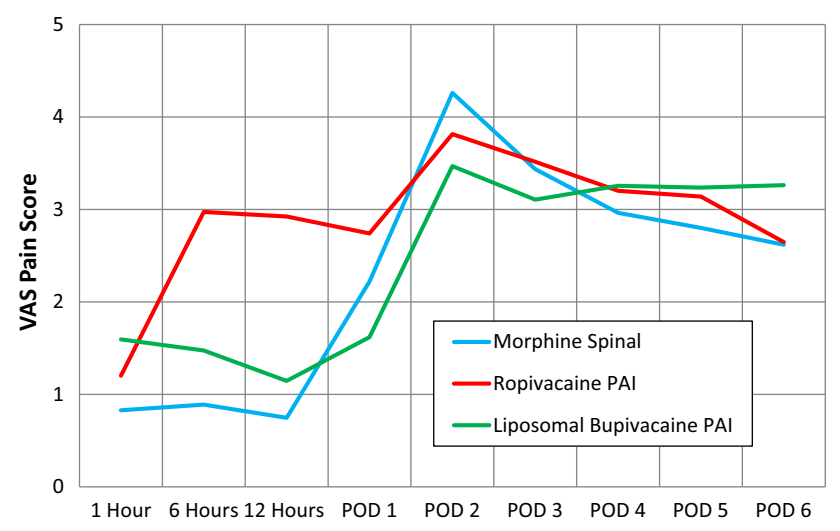

Fig. 2 VAS pain scores are presented for the liposomal bupivacaine PAI, morphine spinal, and ropivacaine PAI groups evaluated at 1,6 , and 12 hours postoperatively and on postoperative days (PODs) 1 through 6 .

transient, because VAS scores by 20-24 hours were no different among the groups. Mean differences in VAS score are viewed with respect to reported thresholds for MCIDs. There is a range of MCIDs reported in the literature, including levels reported by magnitude (from 1.3 to 2.2) $[13,32,33,56,73,81,82]$ and by percentage change in pain $(15 \%-35 \%)[32,33,56,73]$. Although the magnitude of the differences reported here fall within the range of MCIDs reported in the literature, the percentage change is above what could be expected to perceive given the relatively low average pain scores in each group. Patients receiving intrathecal morphine were more likely to experience opioid-associated pruritus, but we could detect no difference in total opioids consumed nor in other adverse reactions.

The primary limitation of the current study is its limited sample size. Although this study had over 100 patients and was well powered to test the hypothesis of lower postoperative pain with liposomal bupivacaine use, much larger sample sizes may be needed to measure differences in opioid consumption and associated adverse events. These adverse events are detrimental to patient rehabilitation and can be very costly to treat [63]. Potentially as a result of the high variability in reported opioid consumption, there were no detectable differences among groups according to this measure. To provide perspective, to detect a doubling of an adverse event that occurs in $20 \%$ of patients would require 164 patients in a two-arm study. Examination of the medians and ranges reported for opioid consumption shows the liposomal bupivacaine PAI group to have a much larger range of opioids consumed, an observation that deserves more consideration in future studies. In addition, there are potential limitations in the generalizability of these results to surgeons who are unfamiliar or unpracticed with some form of PAI technique. In the authors' experience, a moving-needle, multisite injection technique is critically important to the efficacy of liposomal bupivacaine injections. There is also a potential limitation in the amount of Duramorph used in the morphine spinal group. The study protocol was designed for $0.2-0.25 \mathrm{mg}$ to be administered intrathecally, allowing flexibility for patient-specific factors. Although doses up to $0.5 \mathrm{mg}$ have been used for select procedures and patients [30], it is known that adverse events are dose-dependent and some studies recommend using less [14]. There is a possibility that the increase in itching in the spinal morphine group may be a direct result of the morphine dosage. In addition, the reporting of adverse events, including itching, may be subjective, although any cases were referred to the treating nurse, who followed up all reports with a medication and treatment plan. For pain scores, interpretation of results may have benefited from collecting more than a single pain score for each postoperative day after day 1. Lastly, there are potential interactions that may alter the pharmacokinetic or physiochemical properties of liposomal bupivacaine if mixed with other drugs. The PAI cocktail used in this study may be different from that presented previously. Any use of Exparel should be done in accordance with recommendations made on the label.

Previous studies on the efficacy of PAI have generally reported encouraging results, although the study of liposomal bupivacaine specifically has been limited. Crowley et al. [23] conducted a review of studies comparing local anesthetic techniques with placebo injections and intravenous morphine patient-controlled analgesia and found that in most studies (five of six reviewed), local anesthetic techniques reduced opioid use or lowered VAS scores. We previously reported a modest improvement in VAS pain scores, including more pain-free patients, after adding liposomal bupivacaine to a multimodal analgesia regimen for lower extremity TJA [6]. The limitations of that previous study, including the lack of standardized technique between surgeons, confounding medications/anesthesia protocols, and a nonrandomized design, have all been addressed in the current study design. The results of this study are to be contrasted with a retrospective, noncontrolled study by Bagsby et al. [5], which reported that patients injected with liposomal bupivacaine had higher pain scores at discharge compared with those given traditional PAI (ropivacaine, morphine, and epinephrine). In their study, the finding of higher pain in patients given liposomal bupivacaine was observed in the period between the end of the first 24 hours after surgery and discharge. In addition to a lack of randomization, that study did not break down pain scores by day, which is a key limitation because the SD of the length of stay in the traditional PAI injection group was nearly twice that for the liposomal bupivacaine group. Also, as noted in the study, local injection of liposomal 
bupivacaine is technique-dependent, and the authors did not provide details on whether a moving-needle, multisite technique was used. As stated, the authors consider multiple injections essential to the efficacy of the treatment.

Pain control outcomes obtained in the intrathecal morphine group (Group 2) in this study came at a physiological cost: more narcotic-related pruritis (itching) was reported in the intrathecal morphine group than in the other two groups. This is consistent with findings from other authors, including a recent RCT in patients undergoing TKA showing an increased incidence of pruritus (itching) in patients given intrathecal morphine [65]. Many opioid-related adverse events are an immediate health risk and could potentially limit mobility, rehabilitation, and the patient's ability to sleep [61]. Opioid-related adverse events are also reported to increase the use of hospital resources, result in longer hospitalizations, increase costs, and increase the risk for readmission [63]. With the numbers available, total narcotics consumed were not shown to be different among groups. Given the high variability in opioid consumption among patients, additional studies with an increased sample sizes will likely be required. To address this and the other aforementioned limitations, a large multicenter randomized, double-blind study comparing local infiltration with and without liposomal bupivacaine is currently being planned (ClinicalTrials.gov Identifier: NCT02713490).

For the treatments considered in this study, the potential reduction in pain and reduction in itching in the liposomal bupivacaine PAI group should be weighed against the cost (USD 315; data from Pacira Pharmaceuticals). Although the direct costs of treatments in the ropivacaine PAI and spinal morphine groups are lower (both less than approximately USD 20; communication with hospital staff), an in-depth cost analysis will be required to include the effects of patient pain, itching, and other potential adverse events on overall hospital costs.

In summary, this prospective multicenter three-arm blind RCT showed potentially improved pain control at 6 and 12 hours in the liposomal bupivacaine and intrathecal morphine groups compared with the ropivacaine group, and there was a higher risk of pruritus (itching) in the intrathecal morphine group. There were no other differences among the three treatment groups in the early postoperative period. We believe these results highlight the importance of a comprehensive arthroplasty program, which should include incorporating a standard PAI technique regardless of which agents are used and incorporating multimodal analgesia regimens as described. Based on these results, we prefer the use of PAI with liposomal bupivacaine as an alternative to spinal anesthesia with intrathecal morphine as a result of similar postoperative pain control and the potential for reducing adverse events. 


\section{References}

1. Affas F, Nygards EB, Stiller CO, Wretenberg P, Olofsson C. Pain control after total knee arthroplasty: a randomized trial comparing local infiltration anesthesia and continuous femoral block. Acta Orthop. 2011;82:441-447.

2. Asik I, Goktug A, Gulay I, Alkis N, Uysalel A. Comparison of bupivacaine $0.2 \%$ and ropivacaine $0.2 \%$ combined with fentanyl for epidural analgesia during labour. Eur J Anaesthesiol. 2002;19:263-270.

3. Atherton MJ, Feeg VD, el-Adham AF. Race, ethnicity, and insurance as determinants of epidural use: analysis of a national sample survey. Nurs Econ. 2004;22:6-13.

4. Azim SA, Sangster R, Curcio C, Coleman D, Shah U, Zhang S, Reinsel RA, Glass PSA, Nicholson J, Benveniste H. Characterization of Patients with difficult-to-treat acute pain following total knee arthroplasty using multi-modal analgesia. Open Pain J. 2013;6:1-6.

5. Bagsby DT, Ireland PH, Meneghini RM. Liposomal bupivacaine versus traditional periarticular injection for pain control after total knee arthroplasty. J Arthroplasty. 2014;29:1687-1690.

6. Barrington JW, Olugbode O, Lovald S, Ong K, Watson H, Emerson RH. Liposomal bupivacaine: a comparative study of more than 1,000 total joint arthroplasty cases. Orthop Clin North Am. 2015;46:469-477.

7. Beaulieu P, Babin D, Hemmerling T. The pharmacodynamics of ropivacaine and bupivacaine in combined sciatic and femoral nerve blocks for total knee arthroplasty. Anesth Analg. 2006;103:768-774.

8. Beaupre LA, Johnston DB, Dieleman S, Tsui B. Impact of a preemptive multimodal analgesia plus femoral nerve blockade protocol on rehabilitation, hospital length of stay, and postoperative analgesia after primary total knee arthroplasty: a controlled clinical pilot study. Scientific World Journal. 2012;2012:1-6.

9. Bergese SD, Onel E, Morren M, Morganroth J. Bupivacaine extended-release liposome injection exhibits a favorable cardiac safety profile. Reg Anesth Pain Med. 2012;37:145-151.

10. Bergese SD, Ramamoorthy S, Patou G, Bramlett K, Gorfine SR, Candiotti KA. Efficacy profile of liposome bupivacaine, a novel formulation of bupivacaine for postsurgical analgesia. J Pain Res. 2012;5:107-116.

11. Bertini L, Mancini S, Di Benedetto P, Ciaschi A, Martini O, Nava $\mathrm{S}$, Tagariello V. Postoperative analgesia by combined continuous infusion and patient-controlled epidural analgesia (PCEA) following hip replacement: ropivacaine versus bupivacaine. Acta Anaesthesiol Scand. 2001;45:782-785.

12. Bertini L, Tagariello V, Mancini S, Ciaschi A, Posteraro CM, Di Benedetto P, Martini O. $0.75 \%$ and $0.5 \%$ ropivacaine for axillary brachial plexus block: a clinical comparison with $0.5 \%$ bupivacaine. Reg Anesth Pain Med. 1999;24:514-518.

13. Bird SB, Dickson EW. Clinically significant changes in pain along the visual analog scale. Ann Emerg Med. 2001;38:639-643.

14. Bottros MM, Christo PJ. Current perspectives on intrathecal drug delivery. J Pain Res. 2014;7:615-626.

15. Busch CA, Shore BJ, Bhandari R, Ganapathy S, MacDonald SJ, Bourne RB, Rorabeck CH, McCalden RW. Efficacy of periarticular multimodal drug injection in total knee arthroplasty. A randomized trial. J Bone Joint Surg Am. 2006;88:959-963.

16. Capdevila X, Barthelet Y, Biboulet P, Ryckwaert Y, Rubenovitch $J$, d'Athis F. Effects of perioperative analgesic technique on the surgical outcome and duration of rehabilitation after major knee surgery. Anesthesiology. 1999;91:8-15.

17. Carr DB, Reines HD, Schaffer J, Polomano RC, Lande S. The impact of technology on the analgesic gap and quality of acute pain management. Reg Anesth Pain Med. 2005;30:286-291.
18. Cepeda MS, Farrar JT, Baumgarten M, Boston R, Carr DB, Strom BL. Side effects of opioids during short-term administration: effect of age, gender, and race. Clin Pharmacol Ther. 2003;74:102-112.

19. Chen PP, Chui PT, Ma M, Gin T. A prospective survey of patients after cessation of patient-controlled analgesia. Anesth Analg. 2001;92:224-227.

20. Cohen SM. Extended pain relief trial utilizing infiltration of Exparel ${ }^{\mathbb{R}}$, a long-acting multivesicular liposome formulation of bupivacaine: a Phase IV health economic trial in adult patients undergoing open colectomy. J Pain Res. 2012;5:567-572.

21. Coley KC, Williams BA, DaPos SV, Chen C, Smith RB. Retrospective evaluation of unanticipated admissions and readmissions after same day surgery and associated costs. J Clin Anesth. 2002;14:349-353.

22. Crosby E, Sandler A, Finucane B, Writer D, Reid D, McKenna J, Friedlander M, Miller A, O'Callaghan-Enright S, Muir H, Shukla R. Comparison of epidural anaesthesia with ropivacaine $0.5 \%$ and bupivacaine $0.5 \%$ for caesarean section. Can J Anaesth. 1998;45:1066-1071.

23. Crowley C, Dowsey MM, Quinn C, Barrington M, Choong PF. Impact of regional and local anaesthetics on length of stay in knee arthroplasty. ANZ J Surg. 2012;82:207-214.

24. Dasta J, Ramamoorthy S, Patou G, Sinatra R. Bupivacaine liposome injectable suspension compared with bupivacaine $\mathrm{HCl}$ for the reduction of opioid burden in the postsurgical setting. Curr Med Res Opin. 2012;28:1609-1615.

25. Datta S, Camann W, Bader A, VanderBurgh L. Clinical effects and maternal and fetal plasma concentrations of epidural ropivacaine versus bupivacaine for cesarean section. Anesthesiology. 1995;82:1346-1352.

26. Davidson EM, Barenholz Y, Cohen R, Haroutiunian S, Kagan L, Ginosar Y. High-dose bupivacaine remotely loaded into multivesicular liposomes demonstrates slow drug release without systemic toxic plasma concentrations after subcutaneous administration in humans. Anesth Analg. 2010;110:1018-1023.

27. Dihle A, Helseth S, Kongsgaard UE, Paul SM, Miaskowski C. Using the American Pain Society's patient outcome questionnaire to evaluate the quality of postoperative pain management in a sample of Norwegian patients. J Pain. 2006;7:272-280.

28. Dihle A, Helseth S, Paul SM, Miaskowski C. The exploration of the establishment of cutpoints to categorize the severity of acute postoperative pain. Clin J Pain. 2006;22:617-624.

29. Downie WW, Leatham PA, Rhind VM, Wright V, Branco JA, Anderson JA. Studies with pain rating scales. Ann Rheum Dis. 1978;37:378-381.

30. Eandi JA, de Vere White RW, Tunuguntla HS, Bohringer $\mathrm{CH}$, Evans CP. Can single dose preoperative intrathecal morphine sulfate provide cost-effective postoperative analgesia and patient satisfaction during radical prostatectomy in the current era of cost containment? Prostate Cancer Prostatic Dis. 2002;5:226-230.

31. Ethgen O, Bruyere O, Richy F, Dardennes C, Reginster JY. Health-related quality of life in total hip and total knee arthroplasty. A qualitative and systematic review of the literature. J Bone Joint Surg Am. 2004;86:963-974.

32. Farrar JT, Portenoy RK, Berlin JA, Kinman JL, Strom BL. Defining the clinically important difference in pain outcome measures. Pain. 2000;88:287-294.

33. Fishbain DA, Gao J, Lewis JE, Zhang L. At Completion of a multidisciplinary treatment program, are psychophysical variables associated with a VAS improvement of $30 \%$ or more, a minimal clinically important difference, or an absolute VAS score improvement of $1.5 \mathrm{~cm}$ or more? Pain Med. 2016;17:781-789.

34. Freyd M. The graphic rating scale. J Educ Psychol. 1923;14:83102 . 
35. Golf M, Daniels SE, Onel E. A phase 3, randomized, placebocontrolled trial of DepoFoam ${ }^{\circledR}$ bupivacaine (extended-release bupivacaine local analgesic) in bunionectomy. Adv Ther. 2011;28:776-788.

36. Green CR, Anderson KO, Baker TA, Campbell LC, Decker S, Fillingim RB, Kalauokalani DA, Lasch KE, Myers C, Tait RC, Todd $\mathrm{KH}$, Vallerand $\mathrm{AH}$. The unequal burden of pain: confronting racial and ethnic disparities in pain. Pain Med. 2003;4:277-294.

37. Griffin RP, Reynolds F. Extradural anaesthesia for caesarean section: a double-blind comparison of $0.5 \%$ ropivacaine with 0.5\% bupivacaine. Br J Anaesth. 1995;74:512-516.

38. Haas E, Onel E, Miller H, Ragupathi M, White PF. A doubleblind, randomized, active-controlled study for post-hemorrhoidectomy pain management with liposome bupivacaine, a novel local analgesic formulation. Am Surg. 2012;78:574-581.

39. Hall MJ, DeFrances CJ, Williams SN, Golosinskiy A, Schwartzman A. National hospital discharge survey: 2007 summary. Natl Health Stat Report. 2010:1-20, 24.

40. Hayes MHS, Patterson DG. Experimental development of the graphic rating method. Psychol Bull. 1921;18:98-99.

41. Hickey R, Hoffman J, Ramamurthy S. A comparison of ropivacaine $0.5 \%$ and bupivacaine $0.5 \%$ for brachial plexus block. Anesthesiology. 1991;74:639-642.

42. Holzer P. Opioid receptors in the gastrointestinal tract. Regul Pept. 2009;155:11-17.

43. Joshi GP, Cushner FD, Barrington JW, Lombardi AV Jr, Long WJ, Springer BD, Stulberg BN. Techniques for periarticular infiltration with liposomal bupivacaine for the management of pain after hip and knee arthroplasty: a consensus recommendation. J Surg Orthop Adv. 2015;24:27-35.

44. Joyce CR, Zutshi DW, Hrubes V, Mason RM. Comparison of fixed interval and visual analogue scales for rating chronic pain. Eur J Clin Pharmacol. 1975;8:415-420.

45. Kehlet H. Surgical stress: the role of pain and analgesia. $\mathrm{Br} J$ Anaesth. 1989;63:189-195.

46. Kehlet H, Jensen TS, Woolf CJ. Persistent postsurgical pain: risk factors and prevention. Lancet. 2006;367:1618-1625.

47. Kelly AM. Does the clinically significant difference in visual analog scale pain scores vary with gender, age, or cause of pain? Acad Emerg Med. 1998;5:1086-1090.

48. Kerr DR, Kohan L. Local infiltration analgesia: a technique for the control of acute postoperative pain following knee and hip surgery: a case study of 325 patients. Acta Orthop. 2008;79:174183.

49. Kessler ER, Shah M, Gruschkus SK, Raju A. Cost and quality implications of opioid-based postsurgical pain control using administrative claims data from a large health system: opioidrelated adverse events and their impact on clinical and economic outcomes. Pharmacotherapy. 2013;33:383-391.

50. Knudsen K, Beckman Suurkula M, Blomberg S, Sjovall J, Edvardsson N. Central nervous and cardiovascular effects of i.v. infusions of ropivacaine, bupivacaine and placebo in volunteers. Br J Anaesth. 1997;78:507-514.

51. Lansky D, Nwachukwu BU, Bozic KJ. Using financial incentives to improve value in orthopaedics. Clin Orthop Relat Res. 2012;470:1027-1037.

52. Lee A, Fagan D, Lamont M, Tucker GT, Halldin M, Scott DB. Disposition kinetics of ropivacaine in humans. Anesth Analg. 1989;69:736-738.

53. Marcet JE, Nfonsam VN, Larach S. An extended pain relief trial utilizing the infiltration of a long-acting multivesicular liposome formulation of bupivacaine, EXPAREL (IMPROVE): a Phase IV health economic trial in adult patients undergoing ileostomy reversal. J Pain Res. 2013;6:549-555.
54. Marques EM, Blom AW, Lenguerrand E, Wylde V, Noble SM. Local anaesthetic wound infiltration in addition to standard anaesthetic regimen in total hip and knee replacement: long-term cost-effectiveness analyses alongside the APEX randomised controlled trials. BMC Med. 2015;13:151.

55. McGlade DP, Kalpokas MV, Mooney PH, Chamley D, Mark AH, Torda TA. A comparison of $0.5 \%$ ropivacaine and $0.5 \%$ bupivacaine for axillary brachial plexus anaesthesia. Anaesth Intensive Care. 1998;26:515-520.

56. Mease PJ, Spaeth M, Clauw DJ, Arnold LM, Bradley LA, Russell IJ, Kajdasz DK, Walker DJ, Chappell AS. Estimation of minimum clinically important difference for pain in fibromyalgia. Arthritis Care Res. 2011;63:821-826.

57. Moher D, Hopewell S, Schulz KF, Montori V, Gotzsche PC, Devereaux PJ, Elbourne D, Egger M, Altman DG. CONSORT 2010 explanation and elaboration: updated guidelines for reporting parallel group randomised trials. BMJ. 2010;340:1-28.

58. Morrison LM, Emanuelsson BM, McClure JH, Pollok AJ, McKeown DW, Brockway M, Jozwiak H, Wildsmith JA. Efficacy and kinetics of extradural ropivacaine: comparison with bupivacaine. Br J Anaesth. 1994;72:164-169.

59. Morrison RS, Magaziner J, McLaughlin MA, Orosz G, Silberzweig SB, Koval KJ, Siu AL. The impact of post-operative pain on outcomes following hip fracture. Pain. 2003;103:303311.

60. Ng A, Hall, F, Atkinson, A, Kong, K, Hahn, A. Bridging the analgesic gap. Acute Pain. 2000;3:1-6.

61. Oderda G. Challenges in the management of acute postsurgical pain. Pharmacotherapy. 2012;32:6S-11S.

62. Oderda GM, Evans RS, Lloyd J, Lipman A, Chen C, Ashburn M, Burke J, Samore M. Cost of opioid-related adverse drug events in surgical patients. J Pain Symptom Manage. 2003;25:276-283.

63. Oderda GM, Gan TJ, Johnson BH, Robinson SB. Effect of opioid-related adverse events on outcomes in selected surgical patients. J Pain Palliat Care Pharmacother. 2013;27:62-70.

64. Oderda GM, Said Q, Evans RS, Stoddard GJ, Lloyd J, Jackson K, Rublee D, Samore MH. Opioid-related adverse drug events in surgical hospitalizations: impact on costs and length of stay. Ann Pharmacother. 2007;41:400-406.

65. Olive DJ, Barrington MJ, Simone SA, Kluger R. A randomised controlled trial comparing three analgesia regimens following total knee joint replacement: continuous femoral nerve block, intrathecal morphine or both. Anaesth Intensive Care. 2015;43:454-460.

66. Parvataneni HK, Shah VP, Howard H, Cole N, Ranawat AS, Ranawat CS. Controlling pain after total hip and knee arthroplasty using a multimodal protocol with local periarticular injections: a prospective randomized study. J Arthroplasty. 2007;22:33-38.

67. Pavlin DJ, Chen C, Penaloza DA, Buckley FP. A survey of pain and other symptoms that affect the recovery process after discharge from an ambulatory surgery unit. J Clin Anesth. 2004;16:200-206.

68. Perkins FM, Kehlet $\mathrm{H}$. Chronic pain as an outcome of surgery. A review of predictive factors. Anesthesiology. 2000;93:1123-1133.

69. Pogatzki-Zahn EM, Schnabel A, Zahn PK. Room for improvement: unmet needs in postoperative pain management. Exp Rev Neurother. 2012;12:587-600.

70. Practice guidelines for acute pain management in the perioperative setting: an updated report by the American Society of Anesthesiologists Task Force on Acute Pain Management. Anesthesiology. 2012;116:248-273.

71. Preventing Venous Thromboembolic Disease in Patients Undergoing Elective Hip and Knee Arthroplasty: Evidence-Based Guideline and Evidence Report. 2011. Available at: http://www. 
aaos.org/research/guidelines/VTE/VTE_full_guideline.pdf. Accessed December 1, 2015.

72. Richman JM, Liu SS, Courpas G, Wong R, Rowlingson AJ, McGready J, Cohen SR, Wu CL. Does continuous peripheral nerve block provide superior pain control to opioids? A metaanalysis. Anesth Analg. 2006;102:248-257.

73. Salaffi F, Stancati A, Silvestri CA, Ciapetti A, Grassi W. Minimal clinically important changes in chronic musculoskeletal pain intensity measured on a numerical rating scale. Eur J Pain. 2004;8:283-291.

74. Scott DB, Lee A, Fagan D, Bowler GM, Bloomfield P, Lundh R. Acute toxicity of ropivacaine compared with that of bupivacaine. Anesth Analg. 1989;69:563-569.

75. Sharma S, Iorio R, Specht LM, Davies-Lepie S, Healy WL. Complications of femoral nerve block for total knee arthroplasty. Clin Orthop Relat Res. 2010;468:135-140.

76. Sinatra R, Bigham, M. The anatomy and pathophysiology of acute pain. In: Grass J, ed. Problems in Anesthesiology. Philadelphia, PA, USA: Lippincott-Raven; 1997.

77. So JP, Wright JG. The use of three strategies to improve quality of care at a national level. Clin Orthop Relat Res. 2012;470:1006-1016.

78. Southworth S, Peters J, Rock A, Pavliv L. A multicenter, randomized, double-blind, placebo-controlled trial of intravenous ibuprofen 400 and $800 \mathrm{mg}$ every 6 hours in the management of postoperative pain. Clin Ther. 2009;31:1922-1935.

79. Stahmer SA, Shofer FS, Marino A, Shepherd S, Abbuhl S. Do quantitative changes in pain intensity correlate with pain relief and satisfaction? Acad Emerg Med. 1998;5:851-857.

80. Syed HM, Green L, Bianski B, Jobe CM, Wongworawat MD. Bupivacaine and triamcinolone may be toxic to human chondrocytes: a pilot study. Clin Orthop Relat Res. 2011;469:29412947.
81. Todd KH, Funk JP. The minimum clinically important difference in physician-assigned visual analog pain scores. Acad Emerg Med. 1996;3:142-146.

82. Tubach F, Ravaud P, Martin-Mola E, Awada H, Bellamy N, Bombardier C, Felson DT, Hajjaj-Hassouni N, Hochberg M, Logeart I, Matucci-Cerinic M, van de Laar M, van der Heijde D, Dougados M. Minimum clinically important improvement and patient acceptable symptom state in pain and function in rheumatoid arthritis, ankylosing spondylitis, chronic back pain, hand osteoarthritis, and hip and knee osteoarthritis: Results from a prospective multinational study. Arthritis Care Res. 2012;64:1699-1707.

83. Vogel JD. Liposome bupivacaine (EXPAREL ${ }^{\circledR}$ ) for extended pain relief in patients undergoing ileostomy reversal at a single institution with a fast-track discharge protocol: an IMPROVE Phase IV health economics trial. J Pain Res. 2013;6:605-610.

84. Wells GA, Tugwell P, Kraag GR, Baker PR, Groh J, Redelmeier DA. Minimum important difference between patients with rheumatoid arthritis: the patient's perspective. J Rheumatol. 1993;20:557-560.

85. Wheeler M, Oderda GM, Ashburn MA, Lipman AG. Adverse events associated with postoperative opioid analgesia: a systematic review. J Pain. 2002;3:159-180.

86. Writer WD, Stienstra R, Eddleston JM, Gatt SP, Griffin R, Gutsche BB, Joyce TH, Hedlund C, Heeroma K, Selander D. Neonatal outcome and mode of delivery after epidural analgesia for labour with ropivacaine and bupivacaine: a prospective metaanalysis. Br J Anaesth. 1998;81:713-717.

87. Wu CL, Naqibuddin M, Rowlingson AJ, Lietman SA, Jermyn RM, Fleisher LA. The effect of pain on health-related quality of life in the immediate postoperative period. Anesth Analg. 2003;97:1078-1085. 